\title{
Assessment of Condom Utilization among Abobo Woreda, Shubo Kir Kebel, Gambella
}

\author{
Ojulu Olagi* \\ Department of Health, Jimma University, Ethiopia
}

Submission: June 06, 2018; Published: July 03, 2018

*Corresponding author: Ojulu Olagi, Department of Health, Jimma University, Ethiopia, Email: abrishntamirat@gmail.com

\begin{abstract}
Background: Condoms are an integral part of sexual transmitted disease; unwanted pregnancy and human immune efficiency virus (HIV) acquiring immune deficiency syndrome (AIDS) prevention and their use has increased significantly over the past decade.

Objective: The aims of this study are to assess condom utilization among men in Abobo woreda, shubo kir kebel, Gambella.

Method and Material: A cross sectional study design will be employed; the sample will be drawn from systematically selected students. Ethical clearance Jimma University College board and oral consent from study participant will be obtained before commencing to data collection. Data will be collected using questionnaire on socio-demography, knowledge, attitude and preventive practice which was adopted from previous literature.

The data will be checked for completeness before data entry process. Then data analysis will be undergone using SPSS Version 16. Descriptive statistical analysis of frequency, mean and median will be undertaken for continuous variables. Chi-square test will be used to check any association between dependent and independent variables. Binary regression analysis will be employed to identify factors that significantly affect knowledge, attitude and practice with significance level of $\mathrm{p}<0.05$.
\end{abstract}

\section{Introduction}

Acquired immunodeficiency syndrome (AIDS) caused by the human immunodeficiency virus (HIV) is a major health problem in many parts of the world and is considered as a pandemic disease. Sub-Saharan Africa remains the most heavily affected region by HIV. In 2010, about $68 \%$ of all people living with HIV resided in sub- Saharan Africa. It is also accounted for $70 \%$ of new HIV infections, in 2010. Young people ages 15-24 represent $45 \%$ of all new HIV infections. In sub-Saharan Africa, nearly 3.3 million youths are living with HIV. Ethiopia is one of the subSaharan countries highly affected by the HIV/AIDs pandemic. The adult prevalence of HIV infection in Ethiopia was estimated to be $2.4 \%$ in which most of the burden occurring among younger age groups. Adolescence is a transitional phase between childhood and adulthood characterized by physiological, cognitive and emotional changes [1].

The most common changes include developed sexual characteristics, abstract thought, fantasized role in different situations, increased sexual interests and peer influences. HIV/AIDS affects young members of the societies especially adolescents between the age of 15 to 24 who are vulnerable and at risk of the disease. It is also estimated that most regular undergraduate university students lie within the age group of 18 to 24 years. The loss of young adults will certainly affect the overall economic development [2].

According to the fifth report of AIDS in Ethiopia, based on the year 2003 sentinel surveillance findings and estimates of the HIV/AIDS status in the country, the number of people living with HIV/AIDS is about 1.5 million (3.8\% male and 5\%female; $12.6 \%$ urban and $2.6 \%$ rural) out of which about 96000 are children under 15 years. The HIV-infection of children is related to the prevalence rate of HIV/AIDS and mother to child transmission of the virus [3].

Condoms are an integral part of sexual transmitted disease; unwanted pregnancy and human immune efficiency virus (HIV) acquiring immune deficiency syndrome (AIDS) prevention and their use has increased significantly over the past decade. Correct use of condom reduces the risk of HIV transmission by almost 100 percent. Therefore, condom promotion has received considerable attention in the light against the AIDS pandemic. This is particularly important in sub Saharan Africa were HIV transmission is mainly through sexual contact $[4,5]$. 


\section{Statements of the Problem}

There are many problems in the parts of the world; and considered as a pandemic disease. Sub structural Africa remains the most heavily affected region by HIV. In 2010 about $68 \%$ of people living with HIV resided in sub-Saharan Africa. It also accounted for $20 \%$ new HIV infections; in 2010. Young people ages 15-24 represent $45 \%$ of all new HIV infections. In sub Saharan Africa; nearly 3.3 million youths are living with HIV. To sub-Saharan Africa countries are the worst affected worldwide. Under reporting of AIDS case in developing countries including Ethiopia makes the disease spread and mortality increase [6].

Risky sexual behaviors like unprotected sex, multi partnership, no or inconsistence use of condoms and drug abuse are extremely determinate to the health of adolescents putting them at high risk to HIV/AIDS and other sexually transmitted diseases (STDs). It is reasonably possible to think that University students are educated, inspired, able to practice upon the information they receive and as a result, they are among a low risk population. Nevertheless, results of previous studies showed that most sexual risk behaviors among college and University students might have been acquired through a period of Campus life and hence they are likely to be a risk of HIV/AIDS [7]. Therefore, preventing the transmission and the acquisition of HIV must focus upon behavior and behavioral changes. So, it is prudent to conduct the assessment of condom utilization among Jimma University (JU) self-reported sexuality practice.

\section{Review Literature}

\section{Knowledge}

Knowledge of Condom utilization is very important for acquiring optimum health. Moreover, increasing knowledge of HIV/AIDS can be a powerful means of fostering positive attitudes and building safe practices among population. Hence, a clear understanding about knowledge, attitude and practices (KAPs) among any population is very important for planning to control or prevent the spread of HIV [8]

HIV can be transmitted through sharing of meals, clothes and latrines, correct order of condom use and removal of condom from penis after sexual intercourse and protective effect of: correct use of condom, abstains, being faithful to a partner, not having sex with commercial sex workers from HIV transmission, signs and symptoms of HIV infection and duration of antiretroviral treatments [9].

The association on level of education may arise from the increase in knowledge of students and hence increased attitude as they stay in the university is elongated. Knowledgeable students had a favorable attitude regarding HIV/AIDS. Knowledge is an important factor to build positive attitudes towards HIV/AIDS [10].

\section{Attitude}

Attitude towards HIV beliefs in the prevention of HIV, towards the chance of acquiring the virus if his/her sexual partners had multiple partners, towards use of condom is an insult for their partners, towards willingness 80 Wondemagegn Mulu et al.: Knowledge, Attitude and Practices on HIV/AIDS among Students of Jimma University to live in one dormitory with HIV positive students, towards any body's chance to be infected by the virus and towards accessibility of condom when needed $[11,12]$.

University is very high and appreciable. This is interesting and it should be encouraged as the involvement of youths especially students are very important components of the society that will create behavioral change to prevent HIV/AIDs in the community. The student's attitudes towards AIDs were combined with their willingness to live in one dormitory with HIV positive students. The majorities of the students were sympathetic towards AIDS patients' and were against isolating AIDS patients from living in one dormitory. Sex difference by gender showed a significant association with attitude of students towards HIV/AIDs [13].

\section{Practice}

Participants practice on HIV/AIDS addressed to see condom, confident to hold condom on their hand, confident to discuss about condom use by demonstration, unprotected sex, habit of washing genitals after sex, having sex with multiple sexual partners, sex with commercial sex workers, sex after drinking alcohol and after watching porno videos [14]. Having sex without condom, had multiple sexual partners including sex with commercial sex workers (CSWs) indicate such risky behavior can predispose the student's acquisition of HIV. Discus about condom use by demonstration because of religious and socio-cultural norms related to youths [15].

\section{Individual Level Factors Associated with Condom Use}

Much of the quantitative research examining the sexual behavior of African youth has focused on individual-level determinants or correlates of condom use during sexual activity. These include demographic attributes such as age and gender, behavioral characteristics such as engaging in risk taking behavior, and psychosocial factors such as condom use selfefficacy. In this section, I present the existing literature findings on these individual-level factors beginning with the demographic correlates followed by the behavioral and psychosocial factors that have been found to be associated with condom use and sexual activity. The current literature indicates that males report higher condom use than females both in terms of consistent condom use and ever having used condoms [16].

Meekers and Klein (2002) also found gender differences in reported condom use self-efficacy, with significantly higher proportions of males being confident in their ability to negotiate condom use, to use condoms correctly, and to purchase or obtain condoms. These findings are not surprising since the condoms in question are male-controlled contraceptives and prophylactic devices. However, they may also reflect the fact that males have been socialized to exercise authority in sexual decision-making, whereas there are societal expectations of female passivity during sexual encounters [17]. 
MacPhail and Campbell (2001) also found that compared to young females, males tend to externalize the threat of HIV rather than acknowledging personal vulnerability, which may increase the chances that males will fail to engage in safer sex. This, in turn, may mean an increase in female vulnerability to HIV infection in situations characterized by female disempowerment in sexual decision-making.

Findings on the association of age with condom use are mixed. Several studies have found greater odds of condom use with increasing age, while others have found the opposite relationship with older individuals being less likely to use condoms [18]. Two conceivable methodological issues may explain these conflicting findings. The study, the outcome variable was whether a person had ever used condoms, while condom use at last intercourse was the outcome variable in the Camlin and Chibwete study. In the former study, one might expect that older people would have a longer sexual history and, therefore, a higher likelihood of having ever used condoms. On the other hand, when condoms at last intercourse is the outcome variable, younger people may be expected to have a greater likelihood of condom use as they are more likely to be single, a factor found to be associated with greater odds of condom use. In addition, these disparities may reflect differences in the study population such that studies including a wider age group (15-49years), such as the Camlin and Chibwete study, would find that younger people would have higher rates of condom use largely due to their single status. On the other hand, those focusing on the youth (15 - 24 years) would find a positive association between condom use and age [13], as older youth may be less apprehensive about obtaining and using condoms.

There have been some literature documenting that knowledge about HIV and its transmission may be associated with condom use. Higher odds of condom use have been found among those who are knowledgeable about the prophylactic properties of condoms [14] and among those with accurate AIDS-related information, as indicated by the source of AIDS education. This association has held when controlling for education levels and sociodemographic factors [12].

Several researchers have investigated the relationship between people's perceptions of the pros and cons of using condoms, and their reported use of condoms. Two studies that examined the association between perceived benefits of condoms and actual condom use found that perceived benefits were not significant predictors of ever having used condoms and condom use at last intercourse. This is contrary to what would be expected based on the health belief model, which posits that the likelihood of engaging in a health behavior such as condom use is higher if a person perceives high benefits of engaging in the behavior [12].

\section{Distal Context Factors Associated with Condom Use}

Eaton et al. (2003) describe distal context factors as cultural and structural factors, such as poverty, that could potentially affect sexual behavior. As with the proximal context factors described above, distal context factors have tended to be neglected in quantitative research on condom use in Africa. Culture is one distal context factor that could affect condom use refer to culture as a multifaceted entity that includes amongst other things: traditions, societal norms, social discourse, and shared beliefs and practices. As Bruhin (2003) points out, "sexual activity takes place within relationships, social contexts, and cultures" (p. 390); yet, as stated previously, much of the focus in the study of correlates of sexual behavior in the African context has been on individual-level determinants of sexual behavior. As alluded to earlier, many traditional African cultures are patriarchal and women are often socialized to be subservient to men, which could have implications on their ability to negotiate safer sex. also indicate that masculinity in some African societies is associated with unprotected intercourse with multiple partners. Additionally, social norms often discourage young women from carrying condoms because this behavior is misconstrued as an indication of promiscuity [18].

To further complicate the picture, many African populations are facing a changing socio-cultural context that often results in adolescents dealing with contradictory values and fewer guidelines regarding sexuality than in the past because of a shift from the traditional extended family and greater exposure to mass media that often depicts and to some extent "glorifies" casual sexual relationships, often with multiple partners. Urban or rural residence is another distal context factor that could affect condom use rates, for instance, suggest that urban youth might be better informed about HIV/AIDS than their rural peers as a result of higher access to mass media among the latter group.

\section{Factors Distinguishing Condom User Groups}

The use of psychosocial, behavioral, and contextual factors distinguishes among consistent, sporadic and non-condom users. Mahoney et al. (1995) studied the utility of the health belief model, and several behavioral and demographic variables in distinguishing among young American college students who used condoms consistently, occasionally, or never. The results of their study indicated that occasional users were best distinguished from consistent users and nonusers by the number of sexual partners, perceived susceptibility to HIV and STDs, frequency of intoxication during intercourse, perceived susceptibility of one's partner to HIV and STDs, and condom use self-efficacy. Specifically, they found that sporadic condom users were more likely to perceive that they were susceptible to HIV infection or acquiring STDs, [16-18].

\section{Scope of the study}

This study will be conducted among the students of Jimma University third year Economics department. The research will be conducted by extensive distribution of questionnaires and follow up. The scope of the study addressed issues involved with the assessment of condom utilization among Jimma University in the institute of health student. 


\section{Significance of the Study}

Information of the psychosocial determinants of sexual behaviorin Africa and other parts of the developing world is limited and has tended to focus more on individual level determinants than on the more distal cultural, normative, and societal norms that could potentially have a large impact on young people's sexual behaviors including condom use. In addition, there have been no studies to my knowledge that have examined variables that distinguish between different types of condom users among African populations. Therefore, by investigating both individual land distal factors influencing condom use among young people using quantitative methodologies, and by exploring factors that distinguish between different types of condom users, this study fills a gap in our understanding of adolescent sexual behavior. The present study highlights some of the issues that need to be tackled in HIV prevention programs at the individual, University, and community level. It also identifies areas that should be addressed in health and educational policies.

\section{Objective of the Study}

General Objective: To assess condom utilization among Abobo woreda,shubo kir kebel, Gambella.

\section{Specific Objectives:}

a) To evaluate the individual, social, and environmental factors that are associated with actual or intended condom use for HIV prevention among Abobo woreda, shubo kir kebel to identify factors, distinguish consistent, sporadic, and non- condom users in your department.

b) To establish the level of knowledge of HIV/AIDS among Abobo woreda, shubo kir kebel.

c) To examine the attitudes of students towards HIV/AIDS in your department.

d) To identify sexual practices adopted by the students towards HIV/AIDS prevention among your department.

\section{Methods and Materials}

\section{Study Area and Period}

The study will be conducted in Abobo woreda,shubo kir kebel which is found in Gambella, Region The study will be conducted from May to June, 2018.

\section{Study Design}

Community- based descriptive cross-sectional study design will be employed.

\section{Population}

Source Population: All Abobo woreda,shubo kir kebel men in the reproductive age

Study Population: All randomly selected in Abobo woreda, shubo kir kebel

\section{Inclusion and Exclusion Criteria}

Inclusion Criteria: Men in the reproductive age

Exclusion Criteria: Those unable to communicate

\section{Sample Size Determination and Sampling Techniques}

Sample Size Determination: To determine the sample size, the single population proportion formula was used with the necessary assumptions of prevalence (p) 50\% condom use among Abobo woreda, shubo kir kebel with 95\% confidence level, 5\% margin of error.

$$
n=\frac{Z_{\frac{\partial}{2}}^{2} p(1-p)}{d^{2}}=\frac{(1.96)^{2} * 0.5(0.5)}{(0.05)^{2}}=384
$$

Where

$\mathrm{n}=$ the number of regular undergraduate students who took part in the study

$\mathrm{z}=$ standardized normal distribution value at the $95 \% \mathrm{CI}$, which is +1.96

$\mathrm{p}=$ since such type of study is not conducted previously in the area and the proportion of condom use is estimated to be 0.5

$d=$ the margin $5 \%$

i. Considering the multistage nature of sampling technique, a design effect of 2 was used to multiply the sample size as $384^{*} 2=768$

ii. Since the number of regular undergraduate students is $8966(<10,000)$ correction formula was used as:

$$
n_{f}=\frac{n}{1+n / N}=\frac{768}{1+768 / 8966}=707
$$

Considering $10 \%$ non-response rate and the sample size was 778.

\section{Sampling Procedure}

Quantitative research method in which stratified simple random sampling technique will be used to select study subjects from the entire regular under graduate students currently learning in Jimma University. From those colleges the departments will be selected by simple random sampling. The selected departments will be stratified by batch and sex. After proportionally allocating to size, the actual data will be collected from, student's roster which will be used as sampling frame; respondents who will be selected by using simple random sampling from each sex.

\section{Sampling Technique}

Systematic Random Sampling Technique will be used

$$
\mathrm{n}=778 \text { (Male) }
$$

Design effect $=2$ due to two sampling techniques to minimize variability 


\section{Study Variables}

Dependent/ Outcome Variables

Condom Use: Independent / Exposure/explanatory variables

\section{Socio-Economic and Demographic Variables:}
a) Age
b) Sex
c) Religion,
d) Ethnicity
e) Monthly pocket money.
f) Study year
g) Field of study

Attitude toward condom use Behavioral beliefs toward condom use. Knowledge towards the importance of condom use.

\section{Data Processing and Analysis}

The cleaned data will be entered to SPSS version 16.0 software for analysis. The prevalence of condom use determined by taking frequencies and percentage then result will be summarized and

Table 1: Budget requirement for the research paper. presented by tables, charts and graphs. Bivariate association between condom use and socio-demographic characteristics was examined using P. Value $<0.25$. Variables with p-value $<0.25$ in bivariate logistic regression will be added in multivariate logistic regression model with back-ward stepwise method to identify independently associated predictor. The degrees of association between socio-demographic and condom use variables were assessed using AOR and 95\% CI.

\section{Ethical Consideration}

Ethical clearance will be obtained from Jimma University, Institute of Health, Department of Health Behavior and Society. The participants will be asked to sign the consent of participation.

\section{Dissemination Plan}

The result of the study will be presented to Jimma University, Department of Health Behavior and Society. Further attempt will be made to publish it and disseminate to national and international scientific journals.

\section{Research Work Plan}

Budget and Time: All the research work needs time and budget to implement its activities to meet the intended Objectives/ Budget requirement for the research paper Tables $1 \& 2$.

\begin{tabular}{|c|c|c|c|c|c|c|}
\hline \multicolumn{2}{|c|}{ Items } & \multirow{2}{*}{ Nit } & \multirow{2}{*}{ Quantity } & \multicolumn{2}{c|}{ Budget Allocation } & \multirow{2}{*}{ Remark } \\
\cline { 5 - 7 } & & & Unit Cost/birr & Total Cost br & \\
\hline \multirow{2}{*}{ Stationary items } & Paper 5 & packet & 5 & 185 & 925 & \\
\cline { 2 - 7 } & Pen 1 & packet & 1 & 225 & 225 & \\
\hline \multicolumn{2}{|c|}{ Total } & & & & 225 & \\
\hline
\end{tabular}

Table 2: Time Schedule.

\begin{tabular}{|c|c|c|c|c|c|c|c|c|c|c|}
\hline \multirow{2}{*}{ S.No } & \multirow{2}{*}{ Activities/Tasks } & \multicolumn{8}{|c|}{ Month } & \multirow{2}{*}{ Remark } \\
\hline & & March & April & May & June & July & August & September & October & \\
\hline 0 & Problem Identification & & & & & & & & & \\
\hline 1 & Selecting the Research Topics & & & & & & & & & \\
\hline 2 & Literature review & & & & & & & & & \\
\hline 3 & Develop proposal & & & & & & & & & \\
\hline 4 & Submission of $1^{\text {st }}$ draft proposal & & & & & & & & & \\
\hline 5 & Final draft submission & & & & & & & & & \\
\hline 6 & Data collection & & & & & & & & & \\
\hline 7 & Data edition & & & & & & & & & \\
\hline 8 & Data analysis & & & & & & & & & \\
\hline 9 & Data summery write & & & & & & & & & \\
\hline 10 & Submission of essay Report $1^{\text {st }} \mathrm{draft}$ & & & & & & & & & \\
\hline 11 & $\begin{array}{l}\text { Write up finding and incorporating } \\
\text { comment }\end{array}$ & & & & & & & & & \\
\hline 12 & Finalizing the Research Report & & & & & & & & & \\
\hline 13 & $\begin{array}{c}\text { Submission of essay report final } \\
\text { draft }\end{array}$ & & & & & & & & & \\
\hline 14 & Thesis defense & & & & & & & & & \\
\hline
\end{tabular}




\section{Acknowledgment}

We would like to express my profound gratitude and deep appreciation to Jimma University College for this opportunity to carry out this research. We are particularly very grateful for the special assistance of my advisor Mr. Abraham Tamirat for the guidance and direction which he rendered to us throughout the whole time when we are preparing this research proposal.

\section{Refernces}

1. Addis Z, Yalew A, Shiferaw Y, Abebe Alemu, Wubet Birhan, et al. (2013) Knowledge, attitude and practice towards voluntary counseling and testing among university students in Northwest Ethiopia: A cross sectional study. BMC Public Health 13(714): 2-8.

2. Shiferaw Y, Alemu A, Girma A, Afera Getahun, Andarge Kassa, et al. (2011) Assessment of knowledge, attitude and risk behaviors towards HIV/AIDs and other sexual transmitted infections among preparatory students of Gondar town, northwest Ethiopia. BMC Research Notes 4(505): 2-8.

3. Abdool Karim SS, Abdool Karim Q, Preston-Whyte E, Sankar N (1992) Reasons for lack of condom use among high school students. South African Medical Journal 82(2): 107-110.

4. Adih WK, Alexander CS (1999) Determinants of condom use to prevent HIV infection among youth in Ghana. Journal of Adolescent Health 24(1): 63-72.

5. Akwara PA, Madise NJ, Hinde A (2003) Perception of risk of HIV/AIDS and sexual behavior in Kenya. Journal of Biosocial Science 35(3): 385411

6. Bandura A (1994) Social cognitive theory and exercise of control over HIV infection. In RJ DiClemente, JL Peterson (Eds.), Preventing AIDS: Theories and Methods of Behavioral Interventions. New York, USA, p. 25-59.

7. Barker EK, Rich S (1992) Influences on adolescent sexuality in Nigeria and Kenya: Findings from recent focus-group discussions. Studies in Family Planning 23(3): 199-210.
8. Basen-Engquist K, Parcel GS (1992) Attitudes, norms, and self-efficacy: A model of adolescents' HIV-related sexual risk behavior. Health Education Quarterly 19(2): 263-277.

9. Bauni EK, Jarabi BO (2000) Family planning and sexual behavior in the era of HIV/AIDS: The case of Nakuru District, Kenya. Studies in Family Planning 31(1): 69-80.

10. Bauni EK, Jarabi BO (2003) The low acceptability and use of condoms within marriage: Evidence from Nakuru District, Kenya. African Population Studies 18(1): 51-65.

11. Baylies C (2000) Perspectives on gender and AIDS in Africa. In C Baylies, J Bujra, AIDS, Sexuality and Gender in Africa: Collective Strategies and Struggles in Tanzania and Zambia ( $1^{\text {st }}$ edn.); New York, USA, p. 1-24.

12. Becker MG, Barth RP (2000) Power through choices: The development of a sexuality education curriculum for youths in out-of-home care. Child Welfare 79(3): 269-282.

13. Benoit MB (1997) The role of psychological factors on teenagers who become parents out of wedlock. Children and Youth Services Review 19(5-6): 401-413.

14. Ben-Zur H (2003) Peer risk behavior and denial of HIV/AIDS among adolescents. Sex Education 3(1): 75-85.

15. Bertrand JT, Makani B, Djunghu B, Niwembo KL (1991) Sexual behavior and condom use in 10 sites of Zaire. Journal of Sex Research 28(3): 347-364.

16. Blinn-Pike L (1999) Why abstinent adolescents report they have not had sex: Understanding sexually resilient youth. Family Relations 48(3): 295-301.

17. Bruhin E (2003) Power, communication, and condom use: Patterns of HIV-relevant sexual risk management in heterosexual relationship. AIDS Care 15(3): 389-401.

18. Buve A, Bishikwabo-Nsarhaza K, Mutangadura G (2002) The spread and effect of HIV-1 infection in sub-Saharan Africa. The Lancet 359(9322): 2011-2017.

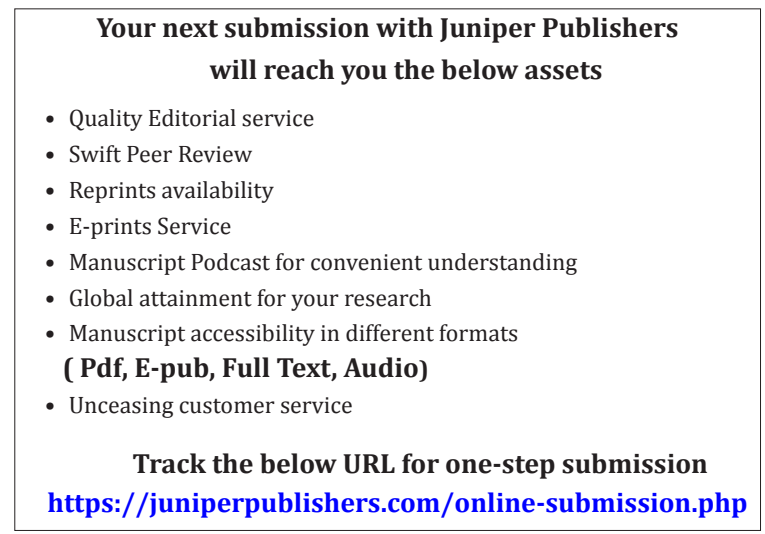

